\title{
3 Research Square

\section{Multimorbidity patterns, polypharmacy and their association with liver and kidney abnormalities in people over 65 years of age: a longitudinal study}

Noemi Villen

Institut Catala De La Salut

Marina Guisado-Clavero

Universitat Autonoma de Barcelona

Sergio Fernández-Bertolín

Universitat Autonoma de Barcelona

Amelia Troncoso

Institut Catala De La Salut; idiap Research Institute

Quinti Foguet-Boreu

Universitat de Vic - Universitat Central de Catalunya

Ester Amado

Idiap Research Institute

Mariona Pons-Vigues

Idiap Research Institute

Albert Roso-Llorach

Idiap Research Institute

Concepción Violán ( $\nabla$ cviolan@idiapjgol.org)

Idiap Research Institute https://orcid.org/0000-0003-3309-5360

Research article

Keywords: Multimorbidity, Epidemiology, Polypharmacy, Primary Health Care, Ageing, Longitudinal study

Posted Date: April 21st, 2020

DOl: https://doi.org/10.21203/rs.3.rs-23421/v1

License: (c) (1) This work is licensed under a Creative Commons Attribution 4.0 International License.

Read Full License

Version of Record: A version of this preprint was published at BMC Geriatrics on June 12th, 2020. See the published version at https://doi.org/10.1186/s12877-020-01580-1. 


\section{Abstract}

Background:

The implementation of individual clinical practice guidelines in patients with multimorbidity often results in polypharmacy. Our aim was to analyse medication use according to longitudinal multimorbidity patterns (MPs) and determine during a 5-year which MPs are associated with abnormal liver and kidney function in primary care patients over 65 years of age living in Catalonia.

Methods:

Design: Longitudinal study (years 2012 to 2016) based on the electronic health records (EHR) contained in the primary care information system database of the Catalan Institute of Health (SIDIAP). Variables : age, sex, MPs, medication and polypharmacy (drug exposure obtained from the Pharmacy Invoice Registry). Medicines were classified in accordance with the Anatomical Therapeutic Chemical Classification System. Glomerular filtration rate was used to determine abnormal kidney function, and serum levels of alkaline phosphatase, alanine transaminase and gamma-glutamyl transpeptidase were used to diagnose abnormal liver function. Statistics : For medication use in MPs, we calculated annual mean packages of each drug in each MP, and observed/expected ratios (O/E-ratios) were obtained by dividing mean packages in the cluster by mean packages of the same drug in the overall population. Logistic regression models were fitted to estimate the association between MPs at baseline and abnormal kidney and liver function tests during follow up.

Results:

916,619 patients were included, and 743,827 completed the follow up. We identified one polypharmacy profile per MP, and concluded that the most prescribed drugs in each pattern corresponded to the diseases overrepresented in that specific MP. The median of drugs ranged from 3 (Cluster 1 - NonSpecific) to 8 (Cluster 10 - Multisystem Pattern). Abnormal kidney function was most commonly observed in the C4-Cardio-Circulatory and Renal (OR 2.19; CI 95\% 2.15-2.23) and C3-Minority Metabolic Autoimmune-Inflammatory (OR 2.16; CI 95\% 2.12-2.20) MPs. A higher risk of abnormal liver function was observed in the C8-Digestive (OR 3.39; CI 95\% 3.30-3.49), and C4-Cardio-Circulatory and Renal (OR 1.96; Cl 95\% 1.91-2.02) MPs.

Conclusions:

A higher risk of abnormal kidney and liver function was observed in specific MPs. The long-term characterisation of MPs and polypharmacy illustrates the burden of chronic multimorbidity and polypharmacy in the elderly population.

\section{Background}


Multimorbidity generally refers to the co-occurrence of multiple chronic medical conditions in a single individual (1). Globally, multimorbidity is growing into a major concern due to the longer lifespan of the population, the complexity of their health status and its relation to greater use of healthcare services ( 2 , 3). Additionally, living with multiple chronic diseases is commonly associated with the use of numerous drugs, function decline, lower of quality of life and increased mortality $(4,5)$.

Generally, prescription in patients with multimorbidity is based on the recommendations of individual disease-specific clinical practice guidelines. This frequently results in polypharmacy (6), commonly defined as the concomitant consumption of 5 or more drugs (7). Polypharmacy is closely related to drugdrug and drug-disease interactions, therapeutic redundancies and adverse drug reactions that can cause hospital admission, worsen chronic conditions and increase morbidity and mortality, especially in the elderly and frail population (8-10). Moreover, many drugs used to treat chronic diseases have a modest benefit at most and an increased risk of adverse reactions in this population.

The dynamic changes in cellular biological processes associated with aging (11) influence the pharmacokinetics and pharmacodynamics of drug metabolization (12). Importantly, the mitochondrial function decline linked to the aging process increases the vulnerability of organs such as heart, lungs, liver and kidney to ischemia/reperfusion injury (13). Consequently, understanding the link between polypharmacy and specific multimorbidity patterns (MPs) and their interrelation with liver and kidney function decline is crucial for the design of strategies that prevent adverse effects of medication among the elderly. Additionally, there is a lack of longitudinal information about the effects of polypharmacy on adverse drug reactions and dynamic cellular changes associated with aging.

The pharmacological management of multimorbidity represents a challenge for health professionals, especially in elderly or frail patients, since it requires a person-centred approach as opposed to treating multiple diseases as independent entities (14). Few publications address medication use according to MPs, and to our knowledge this is the first study which includes long-term data on this issue (15-19).

In another article, we described MPs in people over 65 years of age and followed their trajectories during 5 years. We used soft clustering techniques, which enabled us to study MPs considering as the unit of analysis the patient plus all their diagnosed chronic diseases. We identified multimorbidity trajectories which were mostly stable (20). In addition, we observed that nine over ten patterns involved a large number of chronic diseases and drugs. However, that study did not analyse the characteristics and use of medicines associated with MPs over time, and their association with liver and kidney function abnormalities.

The main aim of this study was to analyse medication use according to longitudinal MPs and characterize them annually in primary care patients over 65 years of age during the 2012-16 period in Catalonia. The secondary aim was to determine which patterns are more likely to present an abnormal liver and kidney function during follow-up. 


\section{Methods}

\section{Design, setting, and study population}

A longitudinal study was carried out in Catalonia (Spain), a Mediterranean region of 7,515,398 inhabitants (2012) (21). The Spanish National Health Service provides universal coverage, financed mainly by tax revenue. The Catalan Health Institute $(\mathrm{CHI})$ manages 285 primary health care centres (PHCCs) in Catalonia that serve $5,501,784$ people, corresponding to the $77.2 \%$ of the population (22).

Inclusion criteria were individuals aged 65-99 years on 31 December 2011 that survived until 31st of December 2012 (index date), with at least one PHCC visit during the 5-year study period (2012-2016). No new entries were allowed in the cohort. Attrition was caused by mortality or transfer to another healthcare provider. We included 916,619 people at baseline and 743,827 completed all follow-up (see Fig. 1).

\section{Data sources}

The Information System for Research in Primary Care (SIDIAP) contains the clinical information from the electronic health records (EHR) of the CHI PHCCs since 2006 (23). The SIDIAP database includes anonymized longitudinal EHR from primary and secondary care that collect information on demographics, symptoms, diagnoses, prescriptions, socio-economic status and the Pharmacy Invoice Registry .

\section{Variables}

All variables were obtained directly from the SIDIAP database (23).

\section{Chronic diseases and multimorbidity}

In the SIDIAP, diseases are coded in accordance with the International Classification of Diseases, version 10 (ICD-10). An operational definition of multimorbidity was used, i.e., the presence of two or more chronic diseases, based on the selected 60 groups of chronic diseases determined by the Swedish National study of Aging and Care in Kungsholmen (24), with additional clinical, laboratory and medication related parameters for the assessment of certain conditions.

\section{Death and drop out}

Death was measured as the occurrence of this event, regardless of cause. If a person transferred out of the $\mathrm{CHI}$ healthcare provider during the study period, they were considered lost to follow-up (drop-outs).

\section{Drugs and polypharmacy}

Information on drug exposure was obtained from the Pharmacy Invoice Registry, which includes drugs prescribed by primary care and hospital physicians and subsidized by the national health system. The study included only systemic drugs, and excluded hospital medication, drugs dispensed by hospital pharmacies, drugs not subsidized by public health services and topical medication (e.g. ointments and 
lotions). Drugs were classified according to the Anatomical Therapeutic Chemical Classification System (ATC) (25). We used the 4th level (Chemical, Pharmacological or Therapeutic subgroup) to facilitate analysis and interpretation. Chronic use was considered when the person used three packages of the drug during each year of the study period. Each drug was coded into a dichotomous variable.

Polypharmacy was defined as the concurrent use of 5 or more drugs in the same individual for each year of the study (7).

\section{Kidney function}

Kidney function was calculated each follow up year using the MDRD-4 IDMS10 equation to estimate glomerular filtration rate (GFR) (26). When one or more GFR was $<60 \mathrm{ml} / \mathrm{min} / 1.73 \mathrm{~m}^{2}$ after the baseline year, we classified it as abnormal kidney function.

\section{Liver function}

Liver function was determined each follow up year by analysing serum levels of alkaline phosphatase (ALP), alanine transaminase (ALT) and gamma-glutamyl transpeptidase (GGT). Liver function was considered abnormal when after baseline, at least one of the values was as follows: ALP $>2 \times 129 \mathrm{IU} / \mathrm{L}$; ALT > 5 × $41 \mathrm{IU} / \mathrm{L}$ (men) or ALT > 5× $33 \mathrm{IU} / \mathrm{L}$ (women); GGT > $61 \mathrm{IU} / \mathrm{L}(27)$.

\section{Other variables}

Demographic variables analysed at baseline and at the end of study were: age at baseline (years), sex (men, women), socio-economic status (MEDEA index; quintiles from least to most deprived) (28) and number of total visits to PHCCs.

\section{Statistical analysis}

Descriptive statistics were used to summarize overall information. Participants' characteristics and prevalence of chronic diseases and medication use were measured at baseline and final year, and during the follow-up period.

MPs were identified using two steps: 1) the dataset was pre-processed by applying a mixture of Principal Component Analysis and Multiple Correspondence Analysis (PCAmix). For the grouping of participants, it was assumed that the patient population was initially distributed into a fuzzy set of clusters, corresponding to the different MPs (29); 2) In order to model the temporal evolution of individuals and clusters, the sequential individual observations were assumed to follow a dynamic random process represented by a Hidden Markov Model (HMM). The set of HMM parameters was fitted to the observation data by applying the Baum-Welch algorithm. Afterwards, a validation process was applied in order to obtain the optimal parameters and longitudinal clusters. The best cluster trajectory is computed by maximizing the probability of the observed sequence, conditioned to the best computed model parameters (Viterbi Algorithm). Next, we associated each cluster with a hidden state or MP to characterize it. 
The final clusters were defined as MPs by the research team based on the clinical significance. We eventually obtained ten MPs, and also drop out and mortality clusters. To describe the MPs, prevalences of diseases in each cluster were calculated. Observed/expected ratios (O/E-ratios) were obtained by dividing disease prevalence in the cluster by disease prevalence in the overall population. Exclusivity, defined as the proportion of patients with the disease included in the cluster over the total number of patients with the disease, was also calculated. A disease was considered to be part of a MP when the $\mathrm{O} / \mathrm{E}$-ratio was $\geq 2$. A more detailed description of the applied machine learning technique can be found elsewhere (30).

\section{Medication use in Multimorbidity patterns}

To describe chronic medication use in each multimorbidity pattern (MP), we calculated annual mean packages for each drug in each cluster. Next, we defined observed/expected ratios (O/E-ratios), obtained by dividing mean packages in the cluster by mean packages in the overall population. We also defined exclusivity as the number of medication packages included in the cluster over the total number of medication packages. A medication was considered part of a MP when the $\mathrm{O} / \mathrm{E}$-ratio was $\geq 2$. As a complementary approach, we also investigated medicines that presented an exclusivity value higher than the percentage frequency of each cluster.

Logistic regression models were fitted to estimate the association between disease clusters (MPs) at baseline and abnormal kidney and liver function values during follow up. Odd ratios (OR) and 95\% confidence intervals $(\mathrm{Cl})$ were adjusted for age, sex, socioeconomic status (MEDEA variable) and total number of packages in 2012. We used multiple imputation to minimize the selection bias resulting from the presence of missing values for MEDEA (7\%). We used multiple imputation by chained equations to obtain seven imputed datasets (31). The final models were fitted with multiple imputed datasets using Rubin's rules to combine effect estimates and standard errors to allow for the uncertainty related to missing data.

The analyses were carried out using R version 3.6.1 (R Foundation for Statistical Computing, Vienna, Austria). The significance level was set at $\mathrm{a}=0.05$.

\section{Results}

A total of 916,619 people over 65 years of age living in Catalonia were included at baseline (women: 57.8\%; mean age: 75.4; standard deviation, SD: 7.4), 853,085 (93.1\%) met multimorbidity criteria, and $487,502(53.2 \%)$ criteria for polypharmacy. At the end of the study period, 743,827 participants had completed follow-up (Fig. 1). The mean number of chronic diseases was 6 (interquartile range (IQR) 4.08.0) at baseline and 7 (IQR 5.0-10.0) in the final year, whereas mean number of medicines during followup was 5 (IQR baseline 2.0-8.0 and IQR at final year 3.0-8.0). The proportion of patients with multimorbidity and polypharmacy was higher at the end of the study period than at baseline $(93.1 \%$ and $53.3 \%$ vs $97.2 \%$ and $56.8 \%$, respectively) (Table 1 ). 
The most prevalent invoiced drugs, from highest to lowest, were proton pump inhibitors (PPI), HMGCoAreductase inhibitors and anilides. During follow-up, the most common drugs remained unchanged, but the prescription had expanded to include more patients (Supplementary File 1).

Ten MPs were identified at baseline (see characteristics of each MP in Table 1). Except for Cluster 1 and Cluster 4, the number of people included in all clusters increased during the study period. Most people remain in the same cluster during follow up. For all MPs, the most common shift is dying. The probability of dying varies significantly depending on which MPs the patient was classified.

\section{Characteristics Of Medication Use In Relation To Multimorbidity Patterns}

Overall, we observed that overrepresented (O/E > 2) drugs (measured as mean packages) in each MP were associated with the overrepresented diseases in that same MP. Naturally, these drugs are intended to treat specific chronic diseases overrepresented in their corresponding MP (Supplementary File 2). For example, in:

\section{Cluster2(C2)-Eye Impairment and Mental}

The most used drugs at baseline were ophthalmologic beta blockers. It should be noted that more than half of the general population with glaucoma and under treatment with ophthalmic beta blockers are included in this cluster (Exclusivity 50.59 and $48.75 \%$ at baseline, respectively).

\section{Cluster3 (C3)-Minority Metabolic Autoimmune-Inflammatory}

At baseline, the most overrepresented drugs were glucocorticoids, uric acid lowering agents, and Vitamin $D$ and analogues.

\section{Cluster4 (C4)-Cardio-Circulatory and Renal}

At baseline, people included in this pattern had 17 different drug groups overrepresented, mainly to treat ischemic cardiovascular disease, heart failure, valvular heart disease, cardiac arrhythmias, diabetes, kidney failure and anaemia (Fig. 3).

Cluster5 (C5)-Cardio-Circulatory, Mental, Respiratory and Genitourinary. At baseline, people included in this pattern had 20 different drug groups overrepresented: peripheral vasodilators, bronchodilators, drugs for ischemic cardiomyopathy, heart failure, prostate diseases and diabetes.

\section{Cluster6 (C6)-Neurological, Digestive and Circulatory}

Overrepresentation in this cluster is mostly constituted by patients with Parkinson's disease and corresponding treatment, mainly dopamine, dopamine derivatives and other medicines used in 
neurological disorders. These diseases and related medications are practically exclusive to this cluster (more than $90.0 \%$ at the beginning of the study).

\section{Cluster7 (C7)-Respiratory and Ear}

Drugs included in this cluster were glucocorticoids, adrenergics in combination with corticosteroids or other drugs excluding anticholinergics, selective beta-2-adrenoreceptor agonists, anticholinergics, corticosteroids, and antihistamines for systemic use.

Cluster8 (C8)-Digestive: The most prescribed medication corresponds to drugs used for the treatment of chronic liver diseases.

Cluster9 (C9)-Neurological, Musculoskeletal and Minor: In this group the most overrepresented drugs are anti-inflammatory and analgesics, in agreement with the MP.

Cluster10 (C10)-Multisystem: The least common pattern, it has multiple overrepresented disorders from different systems, and the corresponding medications used to treat these disorders.

As an example, Fig. 3 represents the evolution during follow up of the O/E ratio and mean number of medication packages for the ten most used drugs in C4-Cardio-Circulatory and Renal and C5-CardioCirculatory, Mental, Respiratory and Genitourinary. In these two MPs, both the O/E ratio and mean number of medication packages increase or remain stable over time.

Considering C1-Non-Specific as a reference, an increase in abnormal kidney function was observed in the different MPs over the study period. The highest proportion of abnormal kidney function was observed in the C4-Cardio-Circulatory and Renal (OR 2.19; $\mathrm{Cl} 95 \%$ 2.15-2.23) and C3-Minority Metabolic AutoimmuneInflammatory MPs (OR 2.16; CI 95\% 2.12-2.20) (Table 2 and Supplement file 3). The MPs with a higher risk of abnormal liver function were C8-Digestive (OR 3.39; Cl 95\% 3.30-3.49), followed by C4-CardioCirculatory and Renal (OR 1.96; Cl 95\% 1.91-2.02) (Table 2 and Supplement File 4).

\section{Discussion}

Key results

This study informs on the use of medication by the elderly population during five years of follow up, according to the ten most common MPs. Predictably, the most overrepresented drugs in each MP coincide with the most overrepresented disorders in that same MP. Also, the medicines most prescribed in the study population remain unchanged throughout the follow up period. The analysis of polypharmacy based on specific MPs and their association with abnormal liver and kidney function has revealed that the patients included in the various MPs present high rates of abnormal liver and kidney function when compared to the MP C1-Non-Specific, underlining the need for new safety criteria in these patients (32, 33). 
In C2-Eye Impairment and Mental, the medicines most frequently prescribed correspond to the ocular disorders diagnosed. However, in other groups of diseases of this MP, for instance the Neurotic, stressrelated and somatoform diseases, which correspond mainly to cigarette consumption, the pharmacological treatment is not subsidised by the public health system and consequently does not appear in the Pharmacy Invoice Registry.

In C3-Minority Metabolic Autoimmune-Inflammatory, over 50\% of diseases are exclusive of this cluster. However, even if the medication is associated with overrepresented conditions, it does not have high exclusivity since these medicines have different authorised indications, for instance glucocorticoids and Vitamin D and analogues, which are even indicated off-label $(34,35)$.

The MPs C4-Cardio-Circulatory and Renal and C5-Cardio-Circulatory, Mental, Respiratory and Genitourinary, illustrate the patients with multimorbidity who are prescribed polypharmacy to treat overrepresented diseases. In addition, some diseases as diabetes that are not overrepresented might need various medicines to control the condition. Moreover, recent intensification of treatments has translated into an increase in drug prescription. $(36,37)$

In C9-Neurological, Musculoskeletal and Minor, metamizole has replaced traditional NSAIDs, probably due to emerging safety concerns of NSAIDs regarding the kidney $(38)$, cardiovascular $(39,40)$, and digestive systems (39).

Abnormal kidney and liver failure function

This study shows that most patients with multimorbidity receiving polypharmacy present abnormal kidney and liver function. While the design of this study cannot explain the causes of the abnormal kidney and liver function observed, we hypothesise that they probably originate from the baseline disorders of the patients, adverse effects from the medication, inappropriate prescribing and lack of adjustment to the kidney and liver function of each patient, which are usually affected by old age (11-13, 41-43). This study, based on medication packages dispensed in pharmacies, underlines the importance of stringent monitoring of prescriptions, particularly in patients that might have abnormal kidney and liver function. We recommend the use of prompts in the electronic health records to adjust medication dosage in accordance with liver function and glomerular filtration rates.

Abnormal kidney function is highest in patients from MPs C4 and C3. In MP C4-Cardio-Circulatory and Renal, in addition to the baseline kidney and cardiovascular disorders of these patients, abnormal kidney function has been attributed to the hemodynamic effects of diuretics causing nephrotoxicity (41). The MP C3-Minority Metabolic Autoimmune-Inflammatory includes patients with hypothyroidism (44) and overrepresentation of allopurinol, both causes of abnormal kidney function.

Abnormal liver function is highest in patients from clusters $\mathrm{C} 8$ and $\mathrm{C} 4$. The risk is highest in patients in C8-Digestive, since this pattern represents patients with liver disease. C4-Cardio-Circulatory and Renal 
has the oldest patients and the highest prescription of vitamin $\mathrm{K}$ antagonists, which can cause cholestasis (42).

Comparison with the literature

Most studies on MPs use a cross-sectional design. Some publications include longitudinal data, but to our knowledge no data on the association of MPs and abnormal kidney and liver function have been published. (45). In the literature, European articles underscore medication for depression and chronic obstructive pulmonary disease (COPD) in the elderly (18), which we included in clusters C9 and C5, respectively. In contrast, Japanese authors observe the highest risk of polypharmacy in malignant, digestive and urologic patterns (17). While we excluded drugs for the treatment of malignancies in our study, we did not observe overrepresentation of drugs for the gastrointestinal system, since they are the type of medication most consumed in the general population, nor for urological diseases, which are highly prevalent in the population over 65 years. Interestingly, in the Japanese study only $25 \%$ of patients were over 65 years of age. Ultimately, if we analysed the drugs overrepresented in specific patterns such as the cardiovascular ( $\mathrm{C} 4$ and $\mathrm{C5}$ ), these medicines would practically replicate polypharmacy patterns described in other publications (18), i.e., drugs for cardiovascular diseases, for diabetes and for gout. However, our medication patterns included also the treatments for other diseases in MPs C4 and C5, for instance anaemia, pain, glaucoma, COPD and benign prostatic hyperplasia.

Strengths and limitations

One of the major strengths of this study is the use of a large, high-quality database that originates from the primary care electronic health records, which includes a large proportion of the population with multimorbidity and with polypharmacy (23). Furthermore, we have used a classification for chronic diseases previously validated by a clinically driven methodology, which allows a homogeneous assessment of chronic diseases and polypharmacy in a manageable number of categories, and also the uniform evaluation of chronicity in the European Union (24).

This study also presents some limitations. Firstly, we only considered the medications for which at least three packages during each year of the study period had been dispensed. While this could underestimate some medicines, it is extremely unusual to dispense less than 3 packages per year of drugs treating chronic diseases. Similarly, we excluded the medication for acute conditions, some of which can cause temporary abnormalities in kidney and liver function. Secondly, the SIDIAP only collects information on drugs prescribed by primary care and hospital physicians which are dispensed by community pharmacies. Consequently, our analysis cannot rule out active ingredients included in hospital drug regimes, over-the-counter medicines and para-pharmacy products, which might also impair kidney and liver function. Thirdly and finally, censoring of people who died and people who transferred might have influenced estimations of risk associations in a competing-risk scenario, which was not an objective of this study. 
Our research shows that while prescription of polypharmacy might be justified with regard to clinical guidelines, it increases the risk of adverse drug reactions and might negatively affect kidney and liver function. Crucially, the risk of overtreatment, which occurs when the prescribed medications have no clinically significant benefit and when the risk of adverse effects associated with an additional medication outweighs the overall benefit of the treatment, is high in polypharmacy $(46,47)$.

\section{Conclusions}

Polypharmacy is highly prevalent in older adults. The most frequently prescribed medicines were related to multimorbidity patterns and their consumption was maintained throughout the follow-up period.

Finally, this study provides real-world data on the evolution of polypharmacy and multimorbidity among older adults and describes the association between polypharmacy and abnormal liver and kidney function in this population.

\section{Declarations}

\section{Ethics approval:}

The protocol of the study was approved by the Clinical Research Ethics Committee, Fundació Institut Universitari per a la recerca a l'Atenció Primària de Salut Jordi Gol i Gurina (IDIAPJGol) (P16/151). All data were anonymized in agreement with national and international law.

\section{Consent for publication}

Not Applicable

\section{Data sharing}

The datasets are not available, as researchers have signed an agreement with the Information System for the Development of Research in Primary Care (SIDIAP) concerning confidentiality and security of the dataset, which forbids providing data to third parties. The SIDIAP is subject to periodic audits.

\section{Competing interests:}

All authors have completed the ICMJE uniform disclosure form at www.icmje.org/coi_disclosure.pdf and declare: no support from any organisation for the submitted work; no financial relationships with any organisations that might have an interest in the submitted work in the previous three years; no other relationships or activities that could have influenced the submitted work.

\section{Funding:}


This work was supported by a research grant from the the Instituto de Salud Carlos III, under the auspices of the Ministry of Science, Innovation and Universities, awarded in the 2016 call under the Health Strategy Action 2013-2016, within the National Research Program oriented to Societal Challenges, within the Technical, Scientific and Innovation Research National Plan 2013-2016 '[grant number PI16/00639]', cofunded with European Union ERDF funds (European Regional Development Fund) and the Department of Health of the Catalan Government, in the 2017 call that awarded subsidies for the Strategic Plan for Research in Health (Pla Estratègic de Recerca i Innovació en Salut, PERIS) 2016-2020, modality research oriented to Primary care '[grant number SLT002/16/00058]' and by the Catalan Government '[grant number AGAUR 2017 SGR 578].

\section{Contributors:}

All authors contributed to the design of the study, revised the article and approved the final version. CV, ARL, MGC, EA and SFB obtained the funding. CV, MGC, QFB, ARL, AT, MPV and NVV drafted the article. NV, $A T, M G, A R, C V$ and SFB proposed the methodology, programmed the tests, and contributed to the analysis and interpretation of data. NV, CV, MGC, and MCB wrote the first draft of the manuscript. NV, AT, $A R L$ and SFB wrote the first draft of the supplementary file, and all authors contributed ideas, interpreted the findings and reviewed rough drafts of the manuscript.

\section{Acknowledgments:}

Not Applicable

\section{References}

1. Valderas JM, Starfi B, Sibbald B. Understanding Health and Health Services. Ann Fam Med. 2009;7(4):357-63.

2. Beard JR, Officer A, de Carvalho IA, Sadana R, Pot AM, Michel J-P, et al. The world report on ageing and health: a policy framework for healthyageing. Lancet. 2016;387(10033):2145-54.

3. Salisbury C, Johnson L, Purdy S, Valderas JM, Montgomery A. Epidemiology and impact of multimorbidity in primary care: a retrospective cohort study. Br J Gen Pract. 2011;61(582):e12-21.

4. Marengoni A, Angleman S, Melis R, Mangialasche F, Karp A, Garmen A, et al. Aging with multimorbidity: A systematic review of the literature. Ageing Res Rev. 2011;10(4):430-9.

5. Violan C, Foguet-Boreu Q, Flores-Mateo G, Salisbury C, Blom J, Freitag M, et al. Prevalence, determinants and patterns of multimorbidity in primary care: A systematic review of observational studies. PLoS One. 2014;9(7):3-11.

6. Guthrie B, Payne K, Alderson P, McMurdo MET, Mercer SW. Adapting clinical guidelines to take account of multimorbidity. BMJ. 2012;345:e6341. 
7. Masnoon N, Shakib S, Kalisch-Ellett L, Caughey GE. What is polypharmacy? A systematic review of definitions. BMC Geriatr. 2017;17:230.

8. Tommelein E, Mehuys E, Petrovic M, Somers A, Colin P, Boussery K. Potentially inappropriate prescribing in community-dwelling older people across Europe: A systematic literature review. Eur $\mathrm{J}$ Clin Pharmacol. 2015;71(12):1415-27.

9. Maher RL, Hanlon J, Hajjar ER. Clinical consequences of polypharmacy in elderly. Expert Opin Drug Saf. 2014;13(1):57-65.

10. Hajjar ER, Cafiero AC, Hanlon JT. Polypharmacy in elderly patients. Am J Geriatr Pharmacother. 2007;5(4):345-51.

11. Ren R, Deng L, Xue Y, Suzuki K, Zhang W, Yu Y, et al. Visualization of aging-associated chromatin alterations with an engineered TALE system. Cell Res. 2017;27(4):483-504.

12. Corsonello A, Pedone C, Incalzi R. Age-Related Pharmacokinetic and Pharmacodynamic Changes and Related Risk of Adverse Drug Reactions. Curr Med Chem. 2010;17(6):571-84.

13. Poulose N, Raju R. Aging and injury: Alterations in cellular energetics and organ function. Aging Dis. 2014;5(2):101-8.

14. Rankin A, Cadogan CA, Patterson SM, Kerse N, Cardwell CR, Bradley MC, et al. Interventions to improve the appropriate use of polypharmacy for older people. Cochrane Database Syst Rev. 2018;9:CD008165.

15. Prados-Torres A, Calderón-Larrañaga A, Hancco-Saavedra J, Poblador-Plou B, Van Den Akker M. Multimorbidity patterns: A systematic review. J Clin Epidemiol. 2014;67(3):254-66.

16. Guisado-Clavero M, Violán C, López-Jimenez T, Roso-Llorach A, Pons-Vigués M, Muñoz MA, et al. Medication patterns in older adults with multimorbidity: A cluster analysis of primary care patients. BMC Fam Pract. 2019;20(1):1-12.

17. Aoki T, Yamamoto Y, Ikenoue T, Onishi Y, Fukuhara S. Multimorbidity patterns in relation to polypharmacy and dosage frequency: A nationwide, cross-sectional study in a Japanese population. Sci Rep. 2018;8(1):3806.

18. Calderón-Larrañaga A, Gimeno-Feliu LA, González-Rubio F, Poblador-Plou B, Lairla-San José M, AbadDíez JM, et al. Polypharmacy patterns: Unravelling systematic associations between prescribed medications. PLoS One. 2013;8(12):e84967.

19. Kostev K, Jacob L. Multimorbidity and polypharmacy among elderly people followed in general practices in Germany. Eur J Intern Med. 2018;55:66-8.

20. Violán C, Fernández-Bertolín S, Guisado-Clavero M, Foguet-Boreu Q, Valderas JM, Vidal J, et al. Longitudinal multimorbidity patterns in elderly population using Hidden Markov Models: a cohort study of Mediterranean elderly population. Sci Rep.

21. Statistical Institute of Catalonia. Idescat. Statistical Yearbook of Catalonia [Internet]. 2016 [cited 2019 Dec 9]. Available from: . 
22. Memòria d'activitats. 2012. Institut Català de la Salut [Internet]. 2012 [cited 2019 Nov 18]. Available from: .

23. García-Gil M, Hermosilla E, Prieto-Alhambra D, Fina F, Rosell M, Ramos R, et al. Construction and validation of a scoring system for the selection of high-quality data in a Spanish population primary care database (SIDIAP). Inform Prim Care. 2011;19(3):135-45.

24. Calderón-Larrañaga A, Vetrano DL, Onder G, Gimeno-Feliu LA, Coscollar-Santaliestra C, Carfí A, et al. Assessing and Measuring Chronic Multimorbidity in the Older Population: A Proposal for Its Operationalization. Journals Gerontol - Ser A Biol Sci Med Sci. 2017;72(10):1417-23.

25. WHO Collaborating Centre for Drug Statistics Methodology. Guidelines for ATC classification and DDD assignment 2013. 16th ed. Oslo; 2013.

26. Elorza-Ricart JM, Tovillas-Morán FJ, Oliveras-Puig A, Galcerán JM, Fina F, Dalfó-Baqué A. Estudio transversal comparativo de las fórmulas CKD-EPI y MDRD-4 a partir de la historia clínica informatizada de Atención Primaria de Barcelona. Hipertens y Riesgo Vasc. 2012;29(4):118-29.

27. Forns J, Cainzos-Achirica M, Hellfritzsch M, Morros R, Poblador-Plou B, Hallas J, et al. Validity of ICD9 and ICD-10 codes used to identify acute liver injury: A study in three European data sources. Pharmacoepidemiol Drug Saf. 2019;28(7):965-75.

28. Domínguez-Berjón MF, Borrell C, Cano-Serral G, Esnaola S, Nolasco A, Pasarín MI, et al. Construcción de un índice de privación a partir de datos censales en grandes ciudades españolas (Proyecto MEDEA). Gac Sanit. 2008;22(3):179-87.

29. Bezdek JC. Pattern Recognition with Fuzzy Objective Function Algorithms. 1st ed. New York. Springer;: Plenum Press; 1981.

30. Rabinier LR. A tutorial on Hidden Markov models and selected applications in speech recognition. Proc IEEE. 1989;77(2):257-86.

31. White IR, Royston P, Wood AM. Multiple imputation using chained equations: Issues and guidance for practice. Stat Med. 2011;30(4):377-99.

32. Duerden M, Avery T, Payne R. Polypharmacy and medicines optimisation Making it safe and sound [Internet]. The King's Fund. London; 2013. Available from: .

33. Cooper JA, Cadogan CA, Patterson SM, Kerse N, Bradley MC, Ryan C, et al. Interventions to improve the appropriate use of polypharmacy in older people: A Cochrane systematic review. BMJ Open. 2015;5:e009235.

34. Morgan DJ, Dhruva SS, Coon ER, Wright SM, Korenstein D. 2018 Update on Medical Overuse. JAMA Intern Med. 2019;179(2):240-6.

35. Woodford HJ, Barrett S, Pattman S. Vitamin D: too much testing and treating? Clin Med (Northfield II). 2018;18(3):196-200.

36. Mata-Cases M, Franch-Nadal J, Real J, Mauricio D. Glycaemic control and antidiabetic treatment trends in primary care centres in patients with type 2 diabetes mellitus during 2007-2013 in Catalonia: A population-based study. BMJ Open. 2016;6(10):4-11. 
37. Lipska KJ, Yao X, Herrin J, McCoy RG, Ross JS, Steinman MA, et al. Trends in drug utilization, glycemic control, and rates of severe hypoglycemia, 2006-2013. Diabetes Care. 2017;40(4):468-75.

38. Dreischulte T, Morales DR, Bell S, Guthrie B. Combined use of nonsteroidal anti-inflammatory drugs with diuretics and/or renin-angiotensin system inhibitors in the community increases the risk of acute kidney injury. Kidney Int. 2015;88(2):396-403.

39. Baigent C, Bhala N, Emberson J, Merhi A, Abramson S, Arber N, et al. Vascular and upper gastrointestinal effects of non-steroidal anti-inflammatory drugs: Meta-analyses of individual participant data from randomised trials. Lancet. 2013;382:769-79.

40. Arfè A, Scotti L, Varas-Lorenzo C, Nicotra F, Zambon A, Kollhorst B, et al. Non-steroidal antiinflammatory drugs and risk of heart failure in four European countries: nested case-control study. BMJ. 2016;354:i4857.

41. Bosch JA, Calbé JJ, Calero F, Díaz JM, Diego L, Esmatjes E, et al. Pautes per a l'harmonització del tractament farmacològic de la malal『a renal crònica. Barcelona: Agència de Qualitat i Avaluació Sanitàries de Catalunya (AQUAS). Departament de Salut. Generalitat de Catalunya; 2016.

42. Morales ML, Vélez L, N, Muñoz MOG. Hepatotoxicidad: Patrón colestásico inducido por fármacos. Rev Colomb Gastroenterol. 2016;31(1):36-47.

43. Tejada F. Hepatotoxicidad por farmacos. Rev Clínica Med Fam. 2010;3(3):177-91.

44. Iglesias P, Díez JJ. Thyroid dysfunction and kidney disease. Eur J Endocrinol. 2009;160(4):503-15.

45. Morin L, Johnell K, Laroche ML, Fastbom J, Wastesson JW. The epidemiology of polypharmacy in older adults: Register-based prospective cohort study. Clin Epidemiol. 2018;10:289-98.

46. Brownlee S, Chalkidou K, Doust J, Elshaug AG, Glasziou P, Heath I, et al. Evidence for overuse of medical services around the world. Lancet. 2017;390:156-68.

47. Benetos A, Rossignol P, Cherubini A, Joly L, Grodzicki T, Rajkumar C, et al. Polypharmacy in the Aging Patient: Management of Hypertension in Octogenarians. J Am Med Assoc. 2015;314(2):170-80.

\section{Tables}

Due to technical limitations, Tables 1-2 are provided in the Supplementary Files section.

\section{Figures}




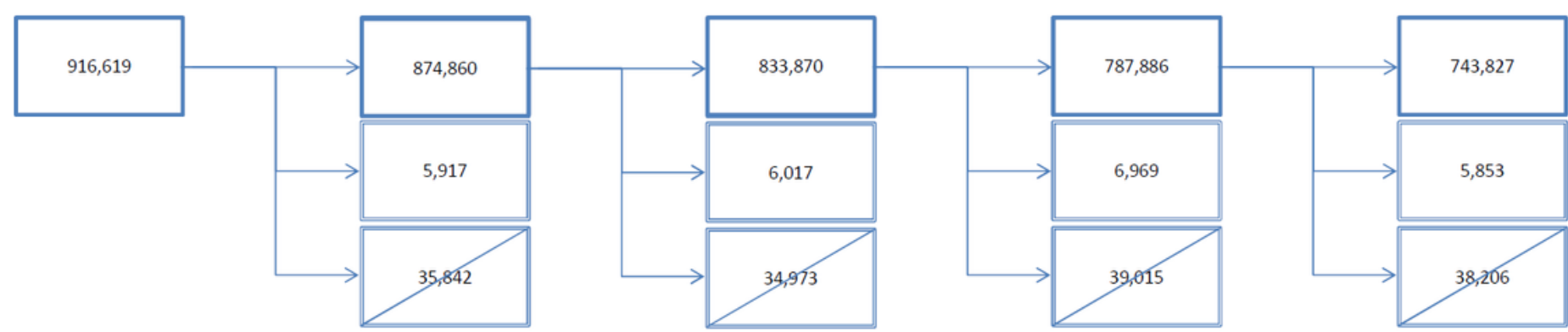

\begin{tabular}{|l||l|}
\hline Alive & Dropped out \\
\hline
\end{tabular}

\section{Figure 1}

\section{Longitudinal Flow Chart of study period (Years 2012-2016; N=916,619 people)}

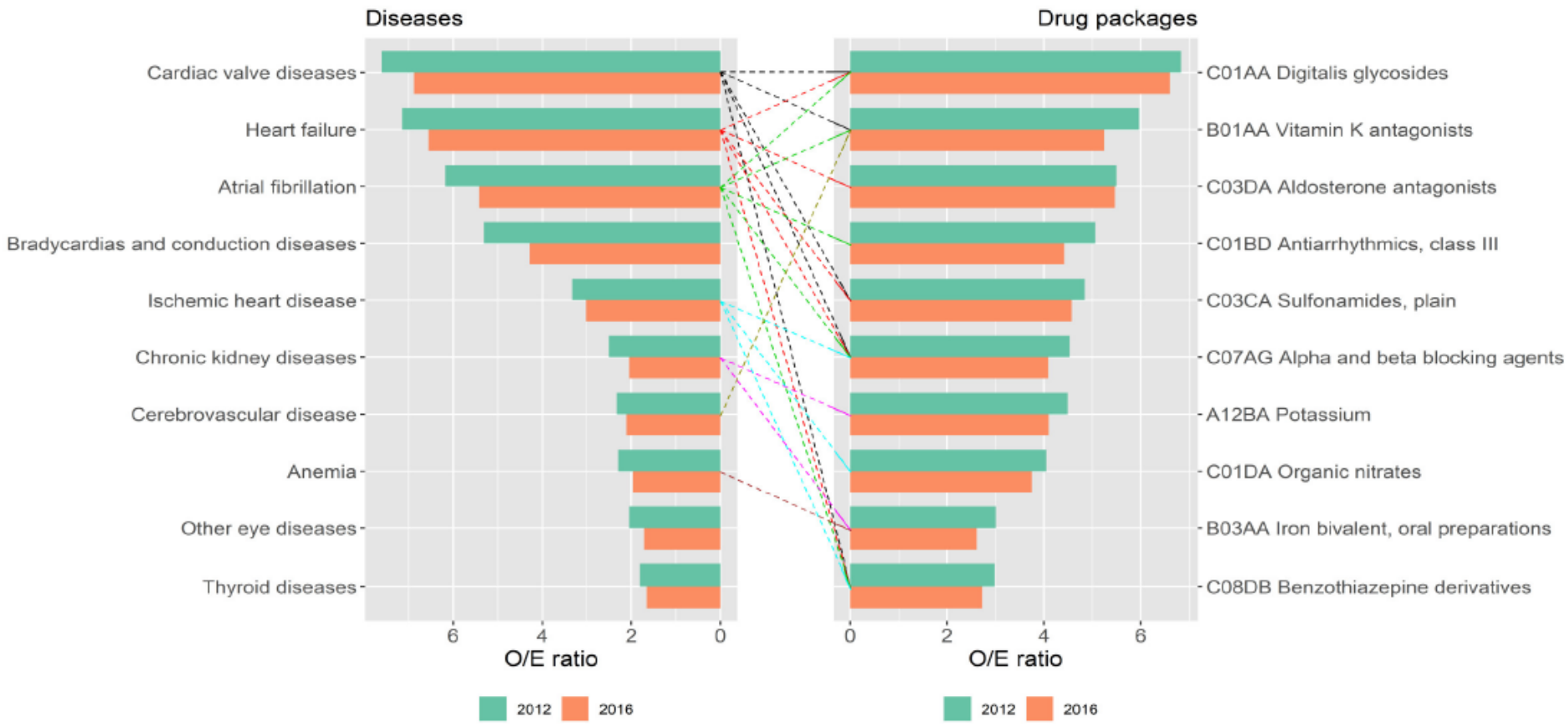

Figure 2

Cardio-Circulatory and Renal patterns in relation with drug prescription 
Cluster 4

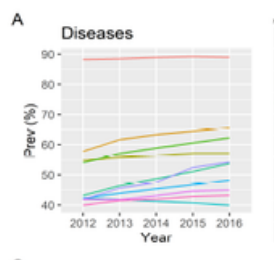

c

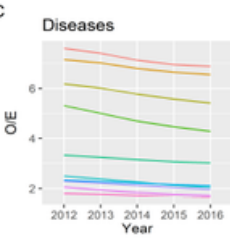

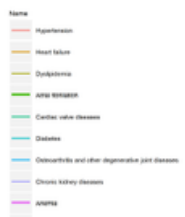
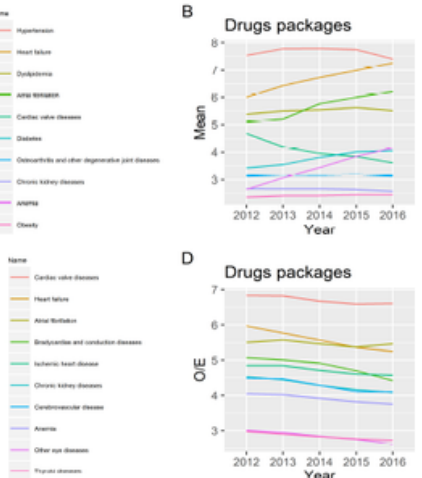

D

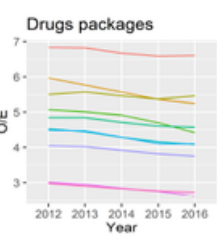

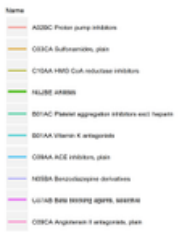

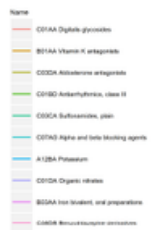

Cluster 5

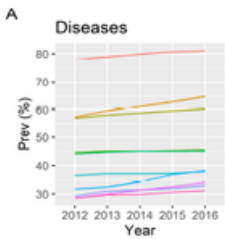

c

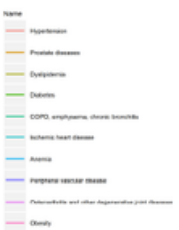

D
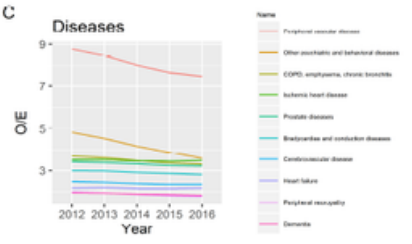
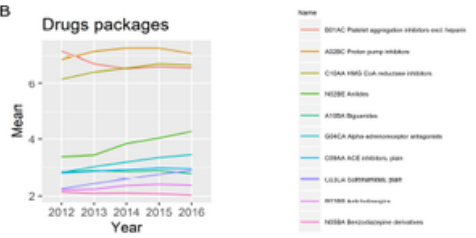

岁.
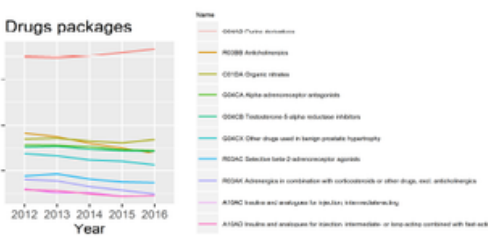

Figure 3

Prevalence and O/E ratio evolution over time for diseases and drugs in Cluster 4 - Cardio Circulatory and Renal and Cluster 5 - Cardio Circulatory, Mental, Respiratory and Genitourinary

\section{Supplementary Files}

This is a list of supplementary files associated with this preprint. Click to download.

- AdditionalFile1.xlsx

- Hepaticvalues5yearssupplementaryfile4.xlsx

- Renalvalues5yearssupplemenmtaryfile3.xlsx

- Table2.pdf

- Table1.pdf

- AdditionalFile2.xlsx 